\title{
Obstructive Sleep Apnea Detection Methods Based on Heart Rate Variability Analysis: Opportunities for a Future Cinc Challenge
}

\author{
Daniele Padovano ${ }^{1}$, Arturo Martínez-Rodrigo ${ }^{1}$, José M Pastor ${ }^{1}$, José J Rieta ${ }^{2}$, Raúl Alcaraz ${ }^{1}$ \\ ${ }^{1}$ Research Group in Electronic, Biomedical and Telecommunications Engineering, \\ University of Castilla-La Mancha, Cuenca, Spain \\ ${ }^{2}$ BioMIT.org, Electronic Engineering Department, Universitat Politecnica de Valencia, Spain
}

\begin{abstract}
The effects of sleep-related disorders, such as obstructive sleep apnea (OSA), can be devastating either in children or adults. Misdiagnosis may lead to severe cardiovascular diseases. Besides, OSA consequences are often related to bad job performance, and road accidents. Nowadays, polysomnography (PSG) is still considered the gold standard for OSA diagnosis, but the required facilities are extremely high, thus reducing availability worldwide. For this reason, simpler and cost-effective diagnosing methods have been proposed in the late years. In this regard, the heart rate variability $(H R V)$ has been demonstrated to strongly reflect apnea episodes during sleep. Hence, this work reviews the latest advances in the evaluation of OSA from the HRV perspective to consider its potentialities for a future revisited CinC Challenge.
\end{abstract}

\section{Introduction}

Sleep apnea syndrome is a disorder in which breathing is repeatedly arrested during sleep. To appraise its severity, an apnea/hypopnea index (AHI) is determined as the number of apnea and hypopnea events per hour of sleep. Prevalence is considered high, ranging from 9 to $38 \%$ (AHI $\leq$ 5 ) in the general population, and it is greater in people suffering from obesity, particularly in men [1]. Two types of sleep apnea syndromes may be found in the literature, i.e., central sleep apnea (CSA) and obstructive sleep apnea (OSA). CSA is characterized by a decrease or abeyance of ventilatory effort, whilst OSA is characterized by repetitive episodes of obstruction of the upper airway [2].

Both varieties are usually associated with a significant decrease in blood oxygen saturation ( $\mathrm{SpO} 2)$ [2]. As a consequence, breathing pauses for a few seconds, which leads to a decrease of blood oxygen and an increase of $\mathrm{CO}_{2}$. This causes the central nervous system activation that results in arousals during sleep [3]. These arousals cease the process of sleep and brings oxygen level back to nor- mal. Furthermore, OSA is often associated with a characteristic snoring pattern that is alternatively followed by silent episodes that usually last 20 to 30 seconds [2]. These episodes, may be noticed by a bed partner. Moreover, arrhythmias and hypoxemia may occur during the aforementioned stages, leading to severe cardiovascular diseases likewise high blood pressure, heart attacks and strokes [4].

Patients suffering from OSA also describe feelings of excessive sleepiness, disorientation, grogginess, and lack of coordination. The daytime sleepiness can be incapacitating, resulting in job loss, road accidents, marital or family problems, and poor school performance [2]. Misdiagnosis can lead patients to being labeled as lazy or as having a primary mental disorder, such as depression. Notwithstanding, polysomnography (PSG) is still currently asserted as the gold standard for OSA diagnosis. PSG recordings typically involve oral-nasal airflow, blood oxygen saturation, chest-abdominal breathing movements, and body position when accompanied with electroencephalogram, electromyogram, and electrocardiogram (ECG). All needed devices put on the patient's body scarcely conveys a reliable sleep. Although this method may provide further information about eventual sleep disorders, its complexity and cost limits the global coverage.

In this regard, heart rate variability (HRV) plays a critical role since it strongly reflects apnea episodes during sleep [5]. In comparison with other magnitudes, such as oral-nasal airflow [6] or the rapid eye movement, HRV is easier to obtain from ECG or photoplethysmography (PPG), and brings accurate results [7]. In the view of more comfortable and cost-effective tools released in the late years, i.e., the single-lead ECG [8] and pulse oximetry devices [9], HRV has become a sort of a new standard in researching. Therefore, because PSG is complex and time-consuming, the search for simpler methods in OSA detection has become a major goal. In this context, this work reviews the latest advances in the evaluation of OSA from the HRV perspective to consider its potentialities for a future revisited CinC Challenge. 


\section{Methodology}

A literature research has been conducted by consulting the following databases: Web of Science, PubMed, Elsevier's ScienceDirect, IEEEXplorer, Springer, and Scopus. The employed key terms were sleep apnea, detection, and heart rate variability. These key terms were combined in advanced searching tools, e.g., "sleep apnea AND heart rate variability" such that 119 articles were found in the period within 2009 and the present. Afterward, a comparison between target key-terms and articles key-terms was performed excluding those articles that mismatched with the topic. Another exclusion criterion was ignoring all articles that were not published in scientific journals, i.e., conferences and congresses. Eventually, 18 articles remained as potential candidates. These articles were fully read and studied as well as some of their inner references. Ultimately, the classification performances and standard procedures were extracted to establish the expected results criteria. However, due to similarities between some methods, only 7 papers were considered in this survey, since they obtained the most promising results in terms of accuracy (Ac), sensitivity (Se), and specificity (Sp).

\section{Sleep apnea detection methods}

In order to organize all the entries of the survey, methods were classified according to the physiological nature of the signal used to extract HRV. Thus, the methods included in this review are based on electrocardiogram (ECG), and pulse oximetry.

\subsection{Methods based on ECG}

In 2018, Zarei and Asl proposed an automatic OSA detection algorithm based on discrete wavelet transform (DWT) and entropy features [10]. They employed two different databases, the Apnea-ECG, from Physiobank [11], and the St. Vicent's University Hospital/University College of Dublin Sleep Apnea Database [12], both available on Physionet's official repository. They segmented the recordings into 60-seconds intervals and then applied DWT to decompose them into several time-frequency scales. Furthermore, they employed complexity measures such as approximate entropy, conditional entropy and similarity, to assess the presence of irregularity within recordings. As a result, 108 features were extracted. Eventually, they applied a sequential feature selection (SFS) algorithm and assessed several machine learning classifiers. In this case, support vector machine (SVM) best performed, obtaining an Ac of $95.7 \%$, Se of $95.8 \%$ and $\mathrm{Sp}$ of $95.6 \%$ as per detected patient, and an Ac of $94.6 \%$, Se of $94.4 \%$, and Sp of $95.7 \%$ as per detected apnea episode during sleep with only 18 features after SFS.
In 2017, Dong et al. developed model based on frequency networks [13]. They also employed the ApneaECG database [11] together with a private dataset. Firstly, they performed a 5-min recording segmentation. Then, they employed the Lomb-Scargle periodogram (LSP) [14] to obtain the power spectral density (PSD) of HRV. The particular point in this work, was the use of dynamic time warping (DTW) to assess similarity between timefrequency series. Thus, a network of HRV segment frequencies was formed up with conventional nodes. Such nodes were connected by means of a threshold value determined by the DTW distance. Thus, as a result of using only 3 network features, they obtained and Ac of $90.1 \%$, Se of $88.3 \%$ and Sp of $90.5 \%$ to detect OSA patients (OSA diagnosis).

In 2016, Cheng et al. conducted a series of experiments in 2-dimensional fractal space based on heterogeneous recurrence analysis [3]. They used the Apnea-ECG database as well [11]. They formed up a state space from the HRV time series using the Takens' delay embedding theorem [15]. The resulting attractor was segmented into multiple sub-regions to facilitate the extraction of local heterogeneous recurrence properties. Moreover, the recurrent patterns between regions were labeled through a multidimensional categorical indexing tree together with a fractal representation of these [16]. Ultimately, a regularized logistic regression model was used to combine the extracted features, thus reaching Ac of 83.0, Se of $83.0 \%$ and $\mathrm{Sp}$ of $82.0 \%$, as per detected apnea episode.

\subsection{Methods based on pulse oximetry}

In July 2019, Bozkurt et al. proposed a method based on PPG by combining multiple machine learning techniques and non-linear features [17]. The initial data was recorded with a PSG device at the Sleep Laboratory of the Department of Pulmonary Diseases at Sakarya Hendek State Hospital. Thereafter, HRV was derived from the PPG peaks, similarly to former ECG recordings. Therefore, they extracted several features from the PPG and the HRV series, which added up to 86 features. Nevertheless, they applied F-score to reduce the number of candidate features. Ultimately, they formed up an ensemble classifier based on the majority vote of several conventional classifiers. Classification results were shown for two different number of selected features. They finally obtained an Ac of 93.0, Se of $93.0 \%$ and $\mathrm{Sp}$ of $96.0 \%$ with only 4 features as per detected patient. It is worth saying that even if apnea was detected, they intended to detect arousals during breathing arrests.

In 2018, Haoyu et al. developed an internet of medical things scheme based on $\mathrm{SpO} 2$ (extracted from PPG) and supported with HRV [18]. In this work, HRV and $\mathrm{SpO} 2$ were both extracted from the St. Vicent's Univer- 
sity/University College of Dublin Apnea database, and a private dataset previously recorded as well. All recordings were scored on a one-minute basis by clinical experts, therefore, these were divided into 1 minute-length segments. In the first place, several statistical features were extracted from HRV and SpO2. In the case of HRV, they extracted the mean, the standard deviation, and the square root of the mean of the sum of the squares pertaining to differences in adjacent RR intervals. Furthermore, they adopted the method proposed by Bsoul et al. [5] to extract the rest of the $\mathrm{SpO} 2$ features. Again, among these, they were the minimum, the variance, the mean, and a correlation coefficient regarding $\mathrm{SpO} 2$ samples for every segment of signal. Eventually, the best performance were obtained employing a SVM approach and using two features from $\mathrm{HRV}$ and other two from $\mathrm{SpO} 2$. As a result, they claimed an Ac of 98.5 , Se of $95.8 \%$ and Sp of $98.9 \%$ as per diagnosed patient.

Also in 2018, Garde et al. developed a pulse oximetrybased OSA screening method [19], which has been validated with the "Sleep" dataset from the British Columbia Children Hospital and a private dataset. All data comprised both PSG and PPG recordings collected at the same time. Firstly, they segmented PPG signals into 2-minute frames. Then, $\mathrm{SpO} 2$ and pulse rate variability (PRV), a surrogate form of HRV, were extracted from every segment. PRV represents a surrogate form of HRV, similarly to what Bozkurt e al. did the following year. In this regard, pulse to pulse intervals (PPI) were obtained by means of a peak detection algorithm. In the frequency domain, each PPI was re-sampled at $4 \mathrm{~Hz}$ to compute its PSD. Regarding SpO2, conventional time-frequency domain parameters were extracted in addition to the delta index, which represents the $\mathrm{SpO} 2$ variability. In this case, they employed multivariate logistic regression models. For each classifier, a stepwise selection method was applied to select the most relevant features and finally train the model. As they assessed classification in terms of AHI, there were different results of Ac for each case. However, for the minimum AHI to be considered apnea (AHI $\geq 5$ ), they obtained Ac of $82.0 \%$, Se of $85.0 \%$ and $\mathrm{Sp}$ of $79.0 \%$ as per diagnosed patient.

In 2015, Garcia-Ravelo et al. introduced a novel approach based on permutation entropy [20]. The CinC Challenge 2000's Database [21] was selected. They conducted signal segmentation with 1-min margin between frames. Then, RR intervals were extracted after an Rpeak detection algorithm followed by an adaptive filtering to remove artifacts. They extracted different features from different analyses based on PE, cepstrum coefficients, and PSD measurements. Regarding PE, the features were extracted by encoding the RR intervals in symbols. Relative frequency was calculated for each symbol and then PE was obtained through its original expression [22]. They

\begin{tabular}{llcc} 
Year & Authors & \# Feat. & Ac (\%) \\
\hline 2019 & Bozkurt et al. [17] & 4 & 93,00 \\
2018 & Zarei and Asl [10] & 18 & 95,71 \\
2018 & Haoyu et al. [18] & 4 & 98,54 \\
2018 & Garde et al. [19] & 5 & 82,00 \\
2017 & Dong et al. [13] & 3 & 90,10 \\
2017 & Martín-Gonzalez et al. [8] & 3 & 84,76 \\
2016 & Cheng et al. [3] & 11 & 85,00 \\
2015 & García-Ravelo et al. [20] & 20 & 84,60 \\
2010 & Bsoul et al. [5] & 111 & 100 \\
2009 & Khandoker et al. [7] & 28 & 92,90 \\
\hline
\end{tabular}

Table 1. Summary of authors and overall accuracy

also derived respiration from the ECG recordings, and then computed PSD. Finally, two classifiers were employed. The best performance was obtained by using 20 features combined with quadratic discriminant analysis, thus obtaining values of $\mathrm{Ac}, \mathrm{Se}$, and $\mathrm{Sp}$ of $84.6 \%, 75.1 \%$, and $90.5 \%$, respectively, as per detected apnea episode.

\section{Conclusions}

The proposed HRV-based OSA detectors have reported promising results, but additional research is still required to consider them as potential alternatives to PSG. In fact, some authors associated HRV features with OSA severity (i.e. the number of apnea episodes per hour) [19,23]. On the other hand, others used HRV features to detect OSA in a minute-by-minute basis [24]. Although accuracy values between 80 and $95 \%$ have been reported in both cases, a high number of HRV features, along with other parameters derived from the ECG, have had to be combined with advanced classifiers. As a reference, former studies have been included in Table 1 to compare the number of employed features in each case. Because short databases have only been used for validation of these methods, more efforts are still required to obtain a realistic view of their generalization capability, and thus of their performance in wider contexts. Consequently, OSA detection is still an interesting opportunity for a future revisited CinC Challenge.

\section{Acknowledgment}

This research has been supported by grants DPI201783952-C3 from MINECO/AEI/FEDER EU, SBPLY/17/ 180501/000411 from Junta de Comunidades de Castilla-la Mancha and AICO/2019/036 from Generalitat Valenciana. Moreover, Daniele Padovano has held graduate research scholarships from Escuela Politécnica de Cuenca and Instituto de Tecnologías Audiovisuales, University of CastillaLa Mancha. 


\section{References}

[1] Senaratna CV, Perret JL, Lodge CJ, Lowe AJ, Campbell BE, Matheson MC, Hamilton GS, Dharmage SC. Prevalence of obstructive sleep apnea in the general population: A systematic review. Sleep Medicine Reviews 2017;34:7081.

[2] Sateia MJ. International Classification of Sleep DisordersThird Edition. Chest 2014;146(5):1387-1394.

[3] Cheng C, Kan C, Yang H. Heterogeneous recurrence analysis of heartbeat dynamics for the identification of sleep apnea events. Computers in Biology and Medicine 2016; 75:10-18

[4] Ghafourian Ms, Tabatabaee Ps, Noori A. Obstructive sleep apnea syndrome diagnosis using HRV signal processing. In 27th Iranian Conference on Electrical Engineering (ICEE). 2019; 1819-1824.

[5] Bsoul M, Minn H, Tamil L. Apnea MedAssist: real-time sleep apnea monitor using single-lead ECG. IEEE Transactions on Information Technology in Biomedicine 2010; 15(3):416-427.

[6] Heitman SJ, Atkar RS, Hajduk EA, Wanner RA, Flemons WW. Validation of nasal pressure for the identification of apneas/hypopneas during sleep. American Journal of Respiratory and Critical Care Medicine 2002;166(3):386-391.

[7] Khandoker AH, Palaniswami M, Karmakar CK. Support vector machines for automated recognition of obstructive sleep apnea syndrome from ECG recordings. IEEE Transactions on Information Technology in Biomedicine 2008; 13(1):37-48.

[8] Martín-González S, Navarro-Mesa JL, Juliá-Serdá G, Kraemer JF, Wessel N, Ravelo-García AG. Heart rate variability feature selection in the presence of sleep apnea: An expert system for the characterization and detection of the disorder. Computers in Biology and Medicine 2017;91:47-58.

[9] Garde A, Dehkordi P, Karlen W, Wensley D, Ansermino JM, Dumont GA. Development of a screening tool for sleep disordered breathing in children using the phone oximeter $^{\mathrm{TM}}$. PloS One 2014;9(11):e112959.

[10] Zarei A, Asl BM. Automatic detection of obstructive sleep apnea using wavelet transform and entropy-based features from single-lead ECG signal. IEEE Journal of Biomedical and Health Informatics 2018;23(3):1011-1021.

[11] Goldberger AL, Amaral LA, Glass L, Hausdorff JM, Ivanov PC, Mark RG, Mietus JE, Moody GB, Peng CK, Stanley HE. PhysioBank, PhysioToolkit, and PhysioNet: components of a new research resource for complex physiologic signals. Circulation 2000;101(23):e215-e220.

[12] McNicholas W, Doherty L, Ryan S, Garvey J, Boyle P, Chua E. St. Vincent's University Hospital / University College Dublin Sleep Apnea Database, 2004. URL https: //physionet.org/content/ucddb/. Type: dataset.

[13] Dong Z, Li X, Chen W. Frequency network analysis of heart rate variability for obstructive apnea patient detection. IEEE Journal of Biomedical and Health Informatics 2017; 22(6):1895-1905.
[14] Lomb NR. Least-squares frequency analysis of unequally spaced data. Astrophysics and Space Science 1976; 39(2):447-462.

[15] Deyle ER, Sugihara G. Generalized theorems for nonlinear state space reconstruction. PLoS One 2011;6(3):e18295.

[16] Yang H, Chen Y. Heterogeneous recurrence monitoring and control of nonlinear stochastic processes. Chaos An Interdisciplinary Journal of Nonlinear Science 2014; 24(1):013138.

[17] Bozkurt MR, Uçar MK, Bozkurt F, Bilgin C. In obstructive sleep apnea patients, automatic determination of respiratory arrests by photoplethysmography signal and heart rate variability. Australasian Physical Engineering Sciences in Medicine 2019;1-21.

[18] Haoyu L, Jianxing L, Arunkumar N, Hussein AF, Jaber MM. An IoMT cloud-based real time sleep apnea detection scheme by using the $\mathrm{SpO} 2$ estimation supported by heart rate variability. Future Generation Computer Systems 2019; 98:69-77.

[19] Garde A, Hoppenbrouwer X, Dehkordi P, Zhou G, Rollinson AU, Wensley D, Dumont GA, Ansermino JM. Pediatric pulse oximetry-based OSA screening at different thresholds of the apnea-hypopnea index with an expression of uncertainty for inconclusive classifications. Sleep Medicine 2019;60:45-52.

[20] Ravelo-García A, Navarro-Mesa J, Casanova-Blancas U, Martin-Gonzalez S, Quintana-Morales P, Guerra-Moreno I, Canino-Rodríguez J, Hernández-Pérez E. Application of the permutation entropy over the heart rate variability for the improvement of electrocardiogram-based sleep breathing pause detection. Entropy 2015;17(3):914-927.

[21] Moody G, Mark R, Goldberger A, Penzel T. Stimulating rapid research advances via focused competition: The Computers in Cardiology Challenge 2000. In Computers in Cardiology, volume 27. 2000; 207-210.

[22] Bandt C, Pompe B. Permutation entropy: a natural complexity measure for time series. Physical Review Letters 2002;88(17):174102.

[23] Garde A, Dekhordi P, Ansermino JM, Dumont GA. Identifying individual sleep apnea/hypoapnea epochs using smartphone-based pulse oximetry. In 38th Annual International Conference of the IEEE Engineering in Medicine and Biology Society (EMBC). 2016; 3195-3198.

[24] Almazaydeh L, Elleithy K, Faezipour M. Obstructive sleep apnea detection using SVM-based classification of ECG signal features. In 34th Annual International Conference of the IEEE Engineering in Medicine and Biology Society (EMBC). 2012; 4938-4941.

Address for correspondence:

Daniele Padovano

Technical School, Campus Universitario, 16071, Cuenca, Spain

Phone: +34-969-179-100

e-mail: daniele.padovano1@alu.uclm.es 